\title{
Glycylglycine plays critical roles in the proliferation of spermatogonial stem cells
}

\author{
BO XU ${ }^{1,2^{*}}$, XIANG WEI $^{1^{*}}$, MINJIAN CHEN ${ }^{1,2^{*}}$, KAIPENG XIE $^{3,4}$, YUQING ZHANG $^{1,2}$, ZHENYAO HUANG $^{1,2}$, \\ TIANYU DONG ${ }^{1,2}$, WEIYUE HU ${ }^{1,2}$, KUN ZHOU $^{1,2}$, XIUMEI HAN ${ }^{1,2}$, XIN WU $^{1}$ and YANKAI XIA ${ }^{1,2}$ \\ ${ }^{1}$ State Key Laboratory of Reproductive Medicine, Institute of Toxicology; ${ }^{2}$ Key Laboratory of Modern Toxicology of \\ Ministry of Education, School of Public Health, Nanjing Medical University, Nanjing, Jiangsu 211166; \\ ${ }^{3}$ Nanjing Maternity and Child Health Care Institute, ${ }^{4}$ Department of Women Health Care, \\ Nanjing Maternity and Child Health Care Hospital, Obstetrics and Gynecology Hospital Affiliated to \\ Nanjing Medical University, Nanjing, Jiangsu 210004, P.R. China
}

Received May 9, 2018; Accepted July 9, 2019

DOI: $10.3892 / \mathrm{mmr} .2019 .10609$

\begin{abstract}
Glial cell line-derived neurotrophic factor (GDNF) is critical for the proliferation of spermatogonial stem cells (SSCs), but the underlying mechanisms remain poorly understood. In this study, an unbiased metabolomic analysis was performed to examine the metabolic modifications in SSCs following GDNF deprivation, and 11 metabolites were observed to decrease while three increased. Of the 11 decreased metabolites identified, glycylglycine was observed to significantly rescue the proliferation of the impaired SSCs, while no such effect was observed by adding sorbitol. However, the expression of self-renewal genes, including B-cell CLL/lymphoma 6 member B, ETS variant 5, GDNF family receptor $\alpha 1$ and early growth response protein 4 remained unaltered following glycylglycine treatment. This finding suggests that although glycylglycine serves an important role in the proliferation of SSCs, it is not required for the self-renewal of SSCs.
\end{abstract}

\section{Introduction}

Spermatogenesis is a complex developmental process that has spermatogonial stem cells (SSCs) at its foundation. The SSCs niche is in the basal compartment of the seminiferous tubules. SSCs undergo spermatogenesis to produce spermatozoa. Self-renewal and the potential to differentiate are two

Correspondence to: Professor Yankai Xia or Professor Xin Wu, State Key Laboratory of Reproductive Medicine, Institute of Toxicology, Nanjing Medical University, 101 Longmian Road, Nanjing, Jiangsu 211166, P.R. China

E-mail: yankaixia@njmu.edu.cn

E-mail: xinwu@njmu.edu.cn

\section{${ }^{*}$ Contributed equally}

Key words: spermatogonial stem cells, glycylglycine, proliferation, metabolomics, glial cell-derived neurotrophic factor properties that distinguish stem cells from somatic cells, and SSCs are the only germline stem cells that can undergo self-renewal division (1). The balance between proliferation and differentiation is therefore essential to the normal function of SSCs and to maintain male fertility (2).

Glial cell line-derived neurotrophic factor (GDNF) is secreted by Sertoli cells, and is an important factor in the cell fate determination of SSCs, which was identified in the year 2000 (3). While $G D N F^{+/}$mice have depleted stem cell reserves (3), the overexpression of GDNF results in the accumulation of undifferentiated spermatogonia and in the development of testicular tumors (4), indicating that the right concentration of GDNF is critical for the proliferation of SSCs (5). Although GDNF has no significant effect on the activity of SSCs at concentrations in the range $1-100 \mathrm{ng} / \mathrm{ml}$, GDNF at concentrations $<1 \mathrm{ng} / \mathrm{ml}$ is insufficient to maintain the proliferation of SSCs in vitro over a period of 7 days (6). However, the mechanisms underlying the GDNF-dependent proliferation of SSCs remain elusive.

Metabolomics is the study of the final products of gene expression and, as a high-throughput analysis, is considered to be more reflective of the biological phenotype compared to genomic, transcriptomic and proteomic studies (7). Thus, metabolomics may be particularly well-suited to detect the dynamic modifications that occur during complex biological processes. Metabolomics has been used in the discovery of small molecules that are potential biomarkers of self-renewal (8), reprogramming (9) and differentiation (10), and it has also been previously used to reveal metabolic mechanisms related to spermatogenesis $(11,12)$.

In the present study, metabolomics was used to evaluate the alterations in SSCs metabolites, including glycylglycine and sorbitol, following GDNF deprivation, in order to elucidate the underlying mechanisms of GDNF-dependent proliferation.

\section{Materials and methods}

Chemicals and reagents. The following chemicals and reagents were purchased from the respective chemical suppliers: Glycylglycine (cat. no. G1002; purity $\geq 99 \%$; Sigma-Aldrich; 
Merck KGaA, Darmstadt, Germany); sorbitol (cat. no. S6021; purity $\geq 98 \%$; Sigma-Aldrich; Merck KGaA); TRIzol ${ }^{\circledR}$ (Invitrogen; Thermo Fisher Scientific, Inc., Waltham, MA, USA); the Cell Counting Kit-8 (CCK-8) assay kit (Beyotime Institute of Biotechnology, Haimen, China); and the cDNA Synthesis kit and SYBR ${ }^{\circledR}$ Green Master Mix kit (Takara Bio, Inc., Otsu, Japan). The Cell Light ${ }^{\mathrm{TM}}$ EdU kit (cat. no. C10310) was purchased from Guangzhou RiboBio Co., Ltd. (Guangzhou, China).

SSCs culture. SSCs were cultured in vitro following a previously described protocol (13), and the cells were characterized as in a previous study (14). All experiments involving mice were approved by the Institutional Animal Care and Use Committee of Nanjing Medical University (IACUC:1601247). The primary cells were isolated from 6-8 day old male C57BL/6 mice obtained from Nanjing Medical University. The mice were housed in groups in a polypropylene cages at $21 \pm 2{ }^{\circ} \mathrm{C}$, a humidity of $50 \pm 10 \%$ and a $12 \mathrm{~h} \mathrm{light/dark} \mathrm{cycle}$ (lights on at 7:00 a.m.), and THY1-positive cells were enriched using magnetic-activated cell separation (Miltenyi Biotech $\mathrm{GmbH}$, Bergisch Gladbach, Germany). Cells were plated at a density of $1.5-2.0 \times 10^{5}$ cells/well on 12-well plates coated with mitotically inactivated SIM mouse embryo-derived thioguanine- and ouabain-resistant feeder layers (cat. no. SNLP76/7-4; American Type Culture Collection, Manassas, VA, USA). Long-term cultures of SSCs were supported in serum-free Minimum Essential Medium- $\alpha$ (Thermo Fisher Scientific, Inc.) and supplied with $20 \mathrm{ng} / \mathrm{ml}$ GDNF (R\&D Systems China Co., Ltd., Shanghai, China), $1 \mathrm{ng} / \mathrm{ml}$ basic fibroblast growth factor 2 (BD Biosciences, San Jose, CA, USA), and $150 \mathrm{ng} / \mathrm{ml}$ GDNF family receptor $\alpha 1$ (GFRA1; R\&D Systems China Co., Ltd.). In addition, $2 \mathrm{mM}$ L-glutamine (Thermo Fisher Scientific, Inc.), $2 \%$ bovine serum albumin, $10 \mu \mathrm{g} / \mathrm{ml}$ transferrin, $50 \mu \mathrm{M}$ free fatty acid mixture $(5.6 \mathrm{mM}$ linolenic acid, $13.4 \mathrm{mM}$ oleic acid, $2.8 \mathrm{mM}$ palmitoleic acid, $35.6 \mathrm{mM}$ linoleic acid, $31 \mathrm{mM}$ palmitic acid, $76.9 \mathrm{mM}$ stearic acid;), $30 \mathrm{nM} \mathrm{Na} \mathrm{SeO}_{3}, 50 \mu \mathrm{M}$ 2-mercaptoethanol, $5 \mu \mathrm{g} / \mathrm{ml}$ insulin, $10 \mathrm{mM}$ 4-(2-hydroxyethyl)-1-piperazineethanesulfonic acid and $60 \mu \mathrm{M}$ putrescine, all purchased from Sigma-Aldrich; Merck KGaA, were added to the medium. Cells were maintained in a humidified atmosphere at $5 \% \mathrm{CO}_{2}$ and $37^{\circ} \mathrm{C}$. The medium was replaced every 2 days and cells were passaged every 5-6 days. For GDNF deprivation, the concentration of GDNF was reduced to $0.1 \mathrm{ng} / \mathrm{ml}$ for $12 \mathrm{~h}$ or $24 \mathrm{~h}$ when SSCs were $\sim 50 \%$ confluent. For the rescue assay, two doses (1 and $10 \mu \mathrm{M}$ ) of each metabolite (glycylglycine and sorbitol) were chosen for optimization (according to http://www.hmdb. ca/metabolites/HMDB0000247). Given results from preliminary studies on the rescue effect, and the glycylglycine and sorbitol level in the body, $10 \mu \mathrm{M}$ was selected for used in the subsequent experiments. After 5 days of treatment, the SSCs were imaged using a light microscope.

Metabolomic analysis. The SSCs sample preparation for metabolomic analysis was conducted according to a previous approach (8). Briefly, treated SSCs were washed five times with ice cold PBS, and $\sim 6 \times 10^{5} \mathrm{SSC} /$ group were collected. Following the addition of $0.3 \mathrm{ml} \mathrm{50 \%} \mathrm{methanol} \mathrm{with} \mathrm{internal}$ standard, the cells were harvested by pipetting. Cells were sonicated for $3 \mathrm{~min}$ (frequency, $20 \mathrm{kHz}$; power, 60\%; pulses, 6/4) and centrifuged at $16,099 \times \mathrm{g}$ at $4^{\circ} \mathrm{C}$ for $15 \mathrm{~min}$ to remove cellular debris. The supernatant was subjected to metabolomic analysis. Quality control (QC) samples were prepared by mixing equal volumes of each SSCs sample.

The metabolomic analysis was performed on an UltiMate ${ }^{\mathrm{TM}}$ 3000 ultra high-performance liquid chromatography system (Dionex; Thermo Fisher Scientific, Inc.), coupled to an Orbitrap high-resolution mass spectrometer (Thermo Fisher Scientific, Inc.) in both positive and negative modes simultaneously (15). The detailed operating procedures was conducted according to a previous study (16). Through this approach, $>70 \%$ of differential metabolites observed in the QC sample had a percent relative standard deviation (\%RSD) of $<30 \%$, and the internal standard had a $\%$ RSD of $<20 \%$, indicating the reliability of the metabolomic analysis (17).

Cell viability assay and proliferation. Cellular viability was evaluated using the CCK-8 Kit. Cells were plated at a density of $1.5 \times 10^{4}$ cells/well in 96-well plates and incubated overnight $\left(37^{\circ} \mathrm{C}, 5 \% \mathrm{CO}_{2}\right)$. The SSCs treatment groups were: Complete medium; GDNF deprivation; GDNF deprivation with glycylglycine rescue; and GDNF deprivation with sorbitol rescue. On the 2nd, 3rd, 4 and 5th days, $10 \mu$ l CCK-8 solution was added to each well, and the cells were incubated for $2 \mathrm{~h}$ at $37^{\circ} \mathrm{C}$ in $5 \%$ $\mathrm{CO}_{2}$. The medium was replaced every 2 days. The absorbance was determined using a TECAN infinite M200 plate reader (Tecan Group, Ltd., Mannedorf, Switzerland) at $450 \mathrm{~nm}$.

The Cell Light ${ }^{\mathrm{TM}}$ EdU kit was used to assess SSCs proliferation. SSCs $\left(\sim 8 \times 10^{3}\right.$ cells/well $)$ were seeded in a 96 -well plate and treated for 5 days as aforementioned. EdU $(25 \mu \mathrm{M})$ was added to the culture medium for an additional $10 \mathrm{~h}$. The cells were fixed with $4 \%$ formaldehyde for $30 \mathrm{~min}$ at room temperature and neutralized using glycine $(2 \mathrm{mg} / \mathrm{ml})$ for $10 \mathrm{~min}$ at room temperature, according to the manufacturer's instructions. Then the cells were permeabilized using $0.5 \%$ Triton X-100 in PBS for $20 \mathrm{~min}$, and staining with Hoechst 33342 was performed. Cell images and data were automatically obtained using High Content Screening (HCS; Cellomics ArrayScan VTI HCS Reader; Thermo Fisher Scientific, Inc.). Appropriate filter sets for the detection of two fluorophores were used, and different fluorescent signals were recorded in two different image collection channels. Channel 1 contained the blue nuclear images; channel 2 contained the red images indicating where EdU was incorporated and which cells were proliferating. For each treatment, three independent wells were measured. The x20 objective was used to collect images, and 48 fields/well were imaged. The analysis was performed after 5 days using the Thermo Scientific HCS Studio Cell Analysis Software 3.0 (Thermo Fisher Scientific, Inc.).

Reverse transcription-quantitative polymerase chain reaction $(R T-q P C R)$ assay of $m R N A$ levels of self-renewal genes. Total RNA was isolated from SSCs using TRIzol ${ }^{\circledR}$ and the concentration was determined using a NanoDrop 2,000 (Thermo Fisher Scientific, Inc., Wilmington, DE, USA). Total RNA $(1 \mu \mathrm{g})$ was used to synthesize cDNA using the following temperature protocol: $37^{\circ} \mathrm{C}$ for $15 \mathrm{~min}$ and $85^{\circ} \mathrm{C}$ for $5 \mathrm{sec}$. The mRNA levels of self-renewal genes, namely B-cell CLL/lymphoma 6 member B (Bcl6b), ETS variant 5 
Table I. Sequences of primers for reverse transcription-quantitative polymerase chain reaction.

\begin{tabular}{lll}
\hline Gene & Type of primer & \multicolumn{1}{c}{ Sequences } \\
\hline Bcl6b & Forward & 5'-GGCTACGTCCGAGAGTTCAC-3' \\
Etv5 & Reverse & 5'-CTTGTGCGCTCTTAGGGGT-3' \\
& Forward & 5'-CACCATGTATCGAGAGGGGC-3' \\
Gfral & Reverse & 5'-GAGCAACCTCTTCCGGTTCT-3' \\
& Forward & 5'-CTCGGAATCCAGCCTACGTC-3' \\
Egr4 & Reverse & $5^{\prime}$-CACTTGTCCTCTCGTGTGCT-3' \\
GAPDH & Forward & 5'-GACGCGCTTCTCTCCAAG-3' \\
& Reverse & 5'-CTCAAAGCCCAGCTCAAGAA-3' \\
& Forward & 5'-AGGTCGGTGTGAACGGATTTG-3' \\
& Reverse & 5'-GGGGTCGTTGATGGCAACA-3'
\end{tabular}

$B c l 6 b, B$-cell CLL/lymphoma 6 member B; Etv5, ETS variant 5; Egr4, early growth response protein 4; Gfral, GDNF family receptor $\alpha 1$.
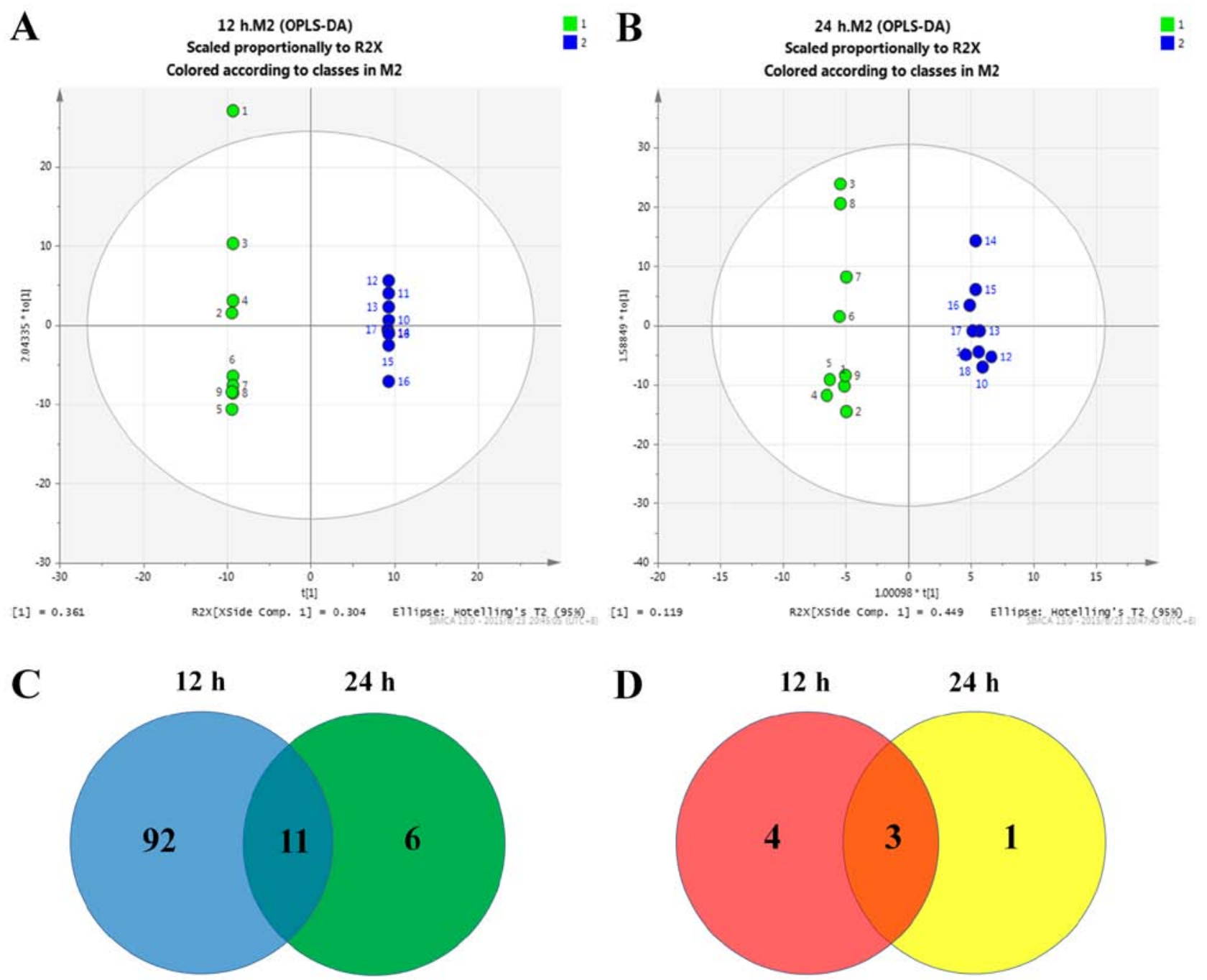

Figure 1. Metabolic modifications after GDNF deprivation. According to the OPLS-DA analysis, obvious separations between samples in the (A) GDNF deprivation for $12 \mathrm{~h}$ and (B) $24 \mathrm{~h}$ groups and the control were detected. (C) A total of 11 metabolites were consistently decreased in both the 12 and $24 \mathrm{~h}$ deprivation groups. (D) A total of three metabolites were increased in both the 12 and $24 \mathrm{~h}$ deprivation groups. OPLS-DA, orthogonal partial least square discriminate analysis; GDNF, glial cell line-derived neurotrophic factor.

(Etv5), Gfral and early growth response protein 4 (Egr4) were analyzed using the SYBR ${ }^{\circledR}$ Green Master Mix kit, according to the manufacturer's instructions. $G A P D H$ was used as a reference gene. Primer sequences synthesized by Invitrogen 
Table II. Altered metabolites following glial cell line-derived neurotrophic factor deprivation for $12 \mathrm{~h}$.

\begin{tabular}{|c|c|c|c|}
\hline Peak & VIP & Fold change & P-value \\
\hline Aminocaproic acid & 1.366 & 0.176 & $3.08 \times 10^{-05}$ \\
\hline Taurine & 1.339 & 0.164 & $5.76 \times 10^{-05}$ \\
\hline Theobromine & 1.330 & 0.178 & $7.06 \times 10^{-05}$ \\
\hline Methylmalonic acid & 1.306 & 0.193 & $1.17 \times 10^{-04}$ \\
\hline Melibiose & 1.301 & 0.107 & $4.86 \times 10^{-05}$ \\
\hline Sucrose & 1.301 & 0.107 & $4.86 \times 10^{-05}$ \\
\hline Trehalose & 1.301 & 0.107 & $4.86 \times 10^{-05}$ \\
\hline Biotin & 1.298 & 0.169 & $1.38 \times 10^{-04}$ \\
\hline Androstenedione & 1.278 & 0.204 & $2.00 \times 10^{-04}$ \\
\hline Petroselinic acid & 1.274 & 0.194 & $2.15 \times 10^{-04}$ \\
\hline Trans-Vaccenic acid & 1.274 & 0.194 & $2.15 \times 10^{-04}$ \\
\hline Indoleacrylic acid & 1.274 & 0.198 & $2.17 \times 10^{-04}$ \\
\hline Sorbitol & 1.269 & 0.195 & $2.37 \times 10^{-04}$ \\
\hline D-Fructose 6-phosphate disodium salt hydrate & 1.259 & 0.236 & $2.85 \times 10^{-04}$ \\
\hline 2-Piperidinemethanol & 1.251 & 0.198 & $3.26 \times 10^{-04}$ \\
\hline Guanine & 1.250 & 0.275 & $3.29 \times 10^{-04}$ \\
\hline Cellobiose & 1.250 & 0.107 & $4.86 \times 10^{-05}$ \\
\hline Formamide & 1.248 & 0.212 & $3.45 \times 10^{-04}$ \\
\hline D-Mannose 6-phosphate sodium salt & 1.242 & 0.393 & $4.84 \times 10^{-02}$ \\
\hline Cinnamaldehyde natural & 1.241 & 0.178 & $3.88 \times 10^{-04}$ \\
\hline Cytosine & 1.229 & 0.200 & $4.70 \times 10^{-04}$ \\
\hline Quinaldic acid & 1.224 & 0.219 & $5.10 \times 10^{-04}$ \\
\hline 16-Alpha-hydroxyestrone & 1.221 & 0.201 & $5.39 \times 10^{-04}$ \\
\hline Androstenediol & 1.221 & 0.201 & $5.39 \times 10^{-04}$ \\
\hline N-Acetyl-L-phenylalanine & 1.221 & 0.201 & $5.39 \times 10^{-04}$ \\
\hline Progesterone & 1.221 & 0.201 & $5.39 \times 10^{-04}$ \\
\hline Uracil & 1.221 & 0.201 & $5.39 \times 10^{-04}$ \\
\hline 2-Amino-1-Phenylethanol & 1.221 & 0.201 & $5.39 \times 10^{-04}$ \\
\hline 2-Deoxycytidine free base & 1.221 & 0.201 & $5.39 \times 10^{-04}$ \\
\hline 4-Hydroxyestrone & 1.221 & 0.201 & $5.39 \times 10^{-04}$ \\
\hline Androsterone & 1.221 & 0.201 & $5.39 \times 10^{-04}$ \\
\hline DL-alpha-Palmitin & 1.221 & 0.201 & $5.39 \times 10^{-04}$ \\
\hline D-Plus-Neopterine & 1.221 & 0.201 & $5.39 \times 10^{-04}$ \\
\hline L-A-Phosphatidylcholine & 1.221 & 0.201 & $5.39 \times 10^{-04}$ \\
\hline Linoleic acid & 1.221 & 0.201 & $5.39 \times 10^{-04}$ \\
\hline 4-Hydroxycinnamic acid & 1.221 & 0.201 & $5.39 \times 10^{-04}$ \\
\hline Acetyl-L-carnitine hydrochloride & 1.221 & 0.201 & $5.39 \times 10^{-04}$ \\
\hline Gamma-Linolenic acid & 1.221 & 0.201 & $5.39 \times 10^{-04}$ \\
\hline Indole-3-acetamide & 1.221 & 0.201 & $5.39 \times 10^{-04}$ \\
\hline Estrone & 1.221 & 0.201 & $5.39 \times 10^{-04}$ \\
\hline Theophylline & 1.221 & 0.201 & $5.39 \times 10^{-04}$ \\
\hline Transtrans-farnesol & 1.221 & 0.201 & $5.39 \times 10^{-04}$ \\
\hline Cytidine & 1.221 & 0.417 & $2.31 \times 10^{-02}$ \\
\hline Riboflavin & 1.218 & 0.202 & $5.62 \times 10^{-04}$ \\
\hline Suberic acid & 1.217 & 0.133 & $5.70 \times 10^{-04}$ \\
\hline 2-methoxycinnamic acid & 1.216 & 0.203 & $5.77 \times 10^{-04}$ \\
\hline N-Acetylglutamine & 1.216 & 0.203 & $5.80 \times 10^{-04}$ \\
\hline 3-Methyladipic acid & 1.215 & 0.203 & $5.93 \times 10^{-04}$ \\
\hline Tyramine & 1.214 & 0.196 & $5.95 \times 10^{-04}$ \\
\hline Octadecanamide & 1.214 & 0.296 & $5.99 \times 10^{-04}$ \\
\hline Guaiacol & 1.213 & 0.204 & $6.06 \times 10^{-04}$ \\
\hline L-Allothreonine & 1.211 & 0.205 & $6.28 \times 10^{-04}$ \\
\hline
\end{tabular}


Table II. Continued.

\begin{tabular}{|c|c|c|c|}
\hline Peak & VIP & Fold change & P-value \\
\hline L-Homoserine & 1.211 & 0.205 & $6.28 \times 10^{-04}$ \\
\hline Octadecanedioic acid & 1.208 & 0.191 & $6.54 \times 10^{-04}$ \\
\hline Gluconolactone & 1.208 & 0.206 & $6.58 \times 10^{-04}$ \\
\hline Sebacic acid & 1.207 & 0.189 & $6.70 \times 10^{-04}$ \\
\hline N-Oleoylethanolamine & 1.206 & 0.207 & $6.78 \times 10^{-04}$ \\
\hline Valeric acid & 1.206 & 0.191 & $6.78 \times 10^{-04}$ \\
\hline Isovaleric acid & 1.206 & 0.198 & $2.73 \times 10^{-04}$ \\
\hline 345-Trimethoxycinnamic acid & 1.204 & 0.208 & $6.98 \times 10^{-04}$ \\
\hline Benzocaine & 1.204 & 0.211 & $6.99 \times 10^{-04}$ \\
\hline 3-(2-Hydroxyethyl)indole & 1.203 & 0.636 & $1.74 \times 10^{-04}$ \\
\hline N-Acetyl-L-alanine & 1.198 & 0.210 & $7.64 \times 10^{-04}$ \\
\hline Folic acid & 1.198 & 0.211 & $7.69 \times 10^{-04}$ \\
\hline D-Glutamic acid & 1.191 & 0.381 & $4.88 \times 10^{-04}$ \\
\hline Hydrocinnamic acid & 1.190 & 0.176 & $8.58 \times 10^{-04}$ \\
\hline 1-Methyl-L-histidine & 1.182 & 0.226 & $9.68 \times 10^{-04}$ \\
\hline 2-Phenylacetamide & 1.177 & 0.379 & $4.75 \times 10^{-05}$ \\
\hline Pentadecanoic acid & 1.171 & 0.221 & $1.13 \times 10^{-03}$ \\
\hline Phthalic acid & 1.169 & 0.081 & $1.01 \times 10^{-02}$ \\
\hline L-Pipecolic acid & 1.169 & 0.267 & $1.16 \times 10^{-03}$ \\
\hline DL-O-Tyrosine & 1.164 & 0.223 & $1.24 \times 10^{-03}$ \\
\hline Glycylglycine & 1.157 & 0.252 & $1.62 \times 10^{-03}$ \\
\hline 3-Ureidopropionic acid & 1.157 & 0.314 & $1.37 \times 10^{-03}$ \\
\hline Thymidine & 1.156 & 0.332 & $1.64 \times 10^{-02}$ \\
\hline Acetoacetic acid lithium & 1.140 & 0.183 & $1.72 \times 10^{-03}$ \\
\hline Betaine & 1.140 & 0.325 & $1.72 \times 10^{-03}$ \\
\hline 5-Phenylvaleric acid & 1.137 & 0.234 & $1.79 \times 10^{-03}$ \\
\hline Rhamnose & 1.135 & 0.282 & $1.83 \times 10^{-03}$ \\
\hline Cinnamic acid & 1.131 & 0.263 & $1.93 \times 10^{-03}$ \\
\hline Acetaminophen & 1.125 & 0.122 & $2.10 \times 10^{-03}$ \\
\hline Acetylglycine & 1.124 & 0.256 & $2.12 \times 10^{-03}$ \\
\hline Cholic acid & 1.115 & 0.244 & $2.37 \times 10^{-03}$ \\
\hline Terephthalic acid & 1.113 & 0.199 & $2.42 \times 10^{-03}$ \\
\hline L-Allo-isoleucine & 1.113 & 0.188 & $2.43 \times 10^{-03}$ \\
\hline Indoleacetic acid & 1.100 & 0.266 & $2.85 \times 10^{-03}$ \\
\hline Cytidine monophosphate & 1.097 & 0.458 & $2.97 \times 10^{-03}$ \\
\hline Glucose 6-phosphate & 1.092 & 0.220 & $3.12 \times 10^{-03}$ \\
\hline Dehydroepiandrosterone & 1.078 & 0.258 & $3.68 \times 10^{-03}$ \\
\hline Mannitol & 1.073 & 0.195 & $2.37 \times 10^{-04}$ \\
\hline Adenine & 1.071 & 0.221 & $3.98 \times 10^{-03}$ \\
\hline Cuminaldehyde & 1.071 & 0.264 & $4.00 \times 10^{-03}$ \\
\hline Trans-Cinnamic acid & 1.067 & 0.274 & $4.18 \times 10^{-03}$ \\
\hline Salicin & 1.063 & 0.264 & $4.37 \times 10^{-03}$ \\
\hline $\mathrm{N}$-Acetylglutamic acid & 1.061 & 0.373 & $4.44 \times 10^{-03}$ \\
\hline Serotonin hydrochloride & 1.061 & 0.265 & $4.46 \times 10^{-03}$ \\
\hline $\mathrm{N}$-Acetylleucine & 1.056 & 0.097 & $4.70 \times 10^{-03}$ \\
\hline Tryptophanol & 1.049 & 0.306 & $5.10 \times 10^{-03}$ \\
\hline Deoxyribose & 1.039 & 0.507 & $3.23 \times 10^{-02}$ \\
\hline 13-Dimethyluracil & 1.037 & 0.287 & $5.77 \times 10^{-03}$ \\
\hline Eicosapentaenoic acid & 1.030 & 0.287 & $6.20 \times 10^{-03}$ \\
\hline L-Aspartyl-L-phenylalanine & 1.018 & 0.320 & $7.04 \times 10^{-03}$ \\
\hline Tryptamine & 1.015 & 0.288 & $7.26 \times 10^{-03}$ \\
\hline Dodecanoic acid & 1.284 & 1.715 & $6.85 \times 10^{-03}$ \\
\hline
\end{tabular}


Table II. Continued.

\begin{tabular}{lccc}
\hline Peak & VIP & Fold change & P-value \\
\hline L-Phenylalanine & 1.284 & 1.885 & $5.32 \times 10^{-04}$ \\
L-Tryptophan & 1.221 & 1.939 & $5.68 \times 10^{-04}$ \\
L-Isoleucine & 1.217 & 2.033 & $1.80 \times 10^{-04}$ \\
L-Leucine & 1.039 & 2.033 & $1.80 \times 10^{-04}$ \\
Inosine & 1.020 & 2.365 & $7.24 \times 10^{-03}$ \\
Ursodeoxycholic acid & 1.015 & 16.743 & $5.65 \times 10^{-03}$ \\
\hline
\end{tabular}

VIP, variable importance in projection.

Table III. Altered metabolites following glial cell line-derived neurotrophic factor deprivation for $24 \mathrm{~h}$.

\begin{tabular}{lccc}
\hline Peak & VIP & Fold change & P-value \\
\hline Glycylglycine & 2.347 & 0.213 & $7.17 \times 10^{-04}$ \\
Sorbitol & 2.109 & 0.527 & $7.00 \times 10^{-04}$ \\
Mannitol & 2.109 & 0.527 & $7.00 \times 10^{-04}$ \\
Biotin & 2.051 & 0.337 & $1.14 \times 10^{-03}$ \\
N-Acetylglutamic acid & 1.913 & 0.430 & $3.13 \times 10^{-03}$ \\
Proline & 1.633 & 0.577 & $1.57 \times 10^{-02}$ \\
Pyrrolidonecarboxylic acid & 1.624 & 0.542 & $1.65 \times 10^{-02}$ \\
Indoleacetic acid & 1.607 & 0.475 & $1.79 \times 10^{-02}$ \\
L-Allothreonine & 1.515 & 0.613 & $2.72 \times 10^{-02}$ \\
L-Homoserine & 1.515 & 0.613 & $2.72 \times 10^{-02}$ \\
D-Mannose 6-phosphate & 1.504 & 0.656 & $2.85 \times 10^{-02}$ \\
sodium salt & & & \\
D-Glutamic acid & 1.498 & 0.531 & $2.93 \times 10^{-02}$ \\
2-Phenylacetamide & 1.498 & 0.425 & $2.93 \times 10^{-02}$ \\
Methyl-L-histidine & 1.477 & 0.475 & $3.21 \times 10^{-02}$ \\
Octadecanedioic acid & 1.149 & 0.488 & $4.27 \times 10^{-02}$ \\
Gluconolactone & 1.147 & 0.516 & $4.39 \times 10^{-02}$ \\
Aminobenzoic acid & 1.137 & 0.363 & $4.98 \times 10^{-02}$ \\
Melibiose & 1.137 & 34.339 & $7.49 \times 10^{-03}$ \\
Sucrose & 1.137 & 34.339 & $7.49 \times 10^{-03}$ \\
Oxidized glutathione & 1.104 & 1.922 & $7.84 \times 10^{-04}$ \\
Inosine & 1.466 & 2.239 & $4.69 \times 10^{-02}$ \\
\hline
\end{tabular}

VIP, variable importance in projection.

(Thermo Fisher Scientific, Inc.) are presented in Table I. All RT-qPCR reactions were performed using the 7900HT Fast Real-Time PCR System (Applied Biosystems; Thermo Fisher Scientific, Inc.) with the following thermocycling conditions: $95^{\circ} \mathrm{C}$ for $30 \mathrm{sec}$ followed by 40 cycles of $95^{\circ} \mathrm{C}$ for $5 \mathrm{sec}$ and $60^{\circ} \mathrm{C}$ for $30 \mathrm{sec}$.

Data analysis. For the metabolomics analysis, the data were imported into SIMCA-P software (Version 13.0; Umetrics; Sartorius AG, Göttingen, Germany) and were unit variance-scaled and auto log-transformed where appropriate. Orthogonal partial least square discriminate analysis (OPLS-DA) was applied to quantitatively assess the metabolites produced by the experimental and control treatments. The metabolites were subsequently indexed by their variable importance in projection (VIP). Data were validated using the leave one out cross-validation method and the quality of model was assessed by R2 and Q2 scores (18). The statistical significance of metabolites identified by OPLS-DA was then calculated via Student's t-test. VIP $>1$ and $\mathrm{P}<0.05$ were considered to indicate a statistically significant difference in metabolite production (19).

The $2^{-\Delta \Delta \mathrm{Cq}}$ method was used to analyze the results of the RT-qPCR (20). Differences among all the treatment groups and the control group were determined by one-way analysis of variance, followed by Dunnett's multiple comparison test. All the statistical analyses were performed using Stata 9.2 (StataCorp LP, College Station, TX, USA) and presented with GraphPad Prism 5 software (GraphPad Software, Inc., La Jolla, CA, USA). All analyses were two-sided and date are presented as the mean $\pm \mathrm{SD}$. $\mathrm{P}<0.05$ was considered to indicate statistically significant difference.

\section{Results}

Metabolic modifications after GDNF deprivation. The OPLS-DA model demonstrated that GDNF deprivation for 12 or $24 \mathrm{~h}$ resulted in a distinct metabolic profile compared to the control $(\mathrm{R} 2 \mathrm{Y}=99.4 \%, \mathrm{Q} 2=71.5 \%$ and $\mathrm{R} 2 \mathrm{Y}=99.0 \%$, $\mathrm{Q} 2=53.0 \%$, respectively; Fig. 1A and B. R2Y indicates the quality of fit, with an R2Y of 1 indicating a perfect description of the data by the model. Q2 indicates the predictive ability of the model, with a Q2 of 1 indicating complete predictability. A total of 103 metabolites had decreased and 7 had increased (VIP>1 and $\mathrm{P}<0.05$ ) after $12 \mathrm{~h}$ of GDNF deprivation (Table II). After $24 \mathrm{~h}$ of GDNF deprivation the majority of those metabolites were restored to normal levels, and only a total of 17 metabolites were decreased (Fig. 1C) and 4 were increased (Fig. 1D; Table III). A comparison of these two lists demonstrated that there were 14 overlapping metabolites. Among these, based on the metabolic information in the HMDB database, glycylglycine and sorbitol were found to be the two most significantly decreased metabolites following GDNF deprivation. Therefore, the effects of glycylglycine and sorbitol on the proliferation of SSCs were further assessed. 
A

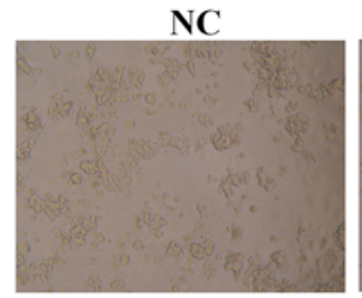

GDNF-0.1

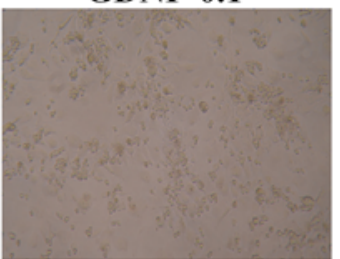

GDNF-0.1+G

GDNF-0.1+S

B

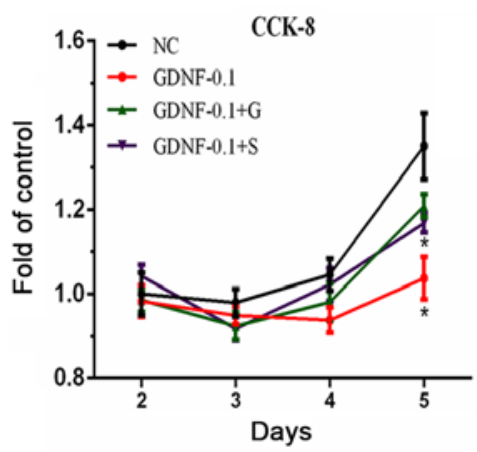

C

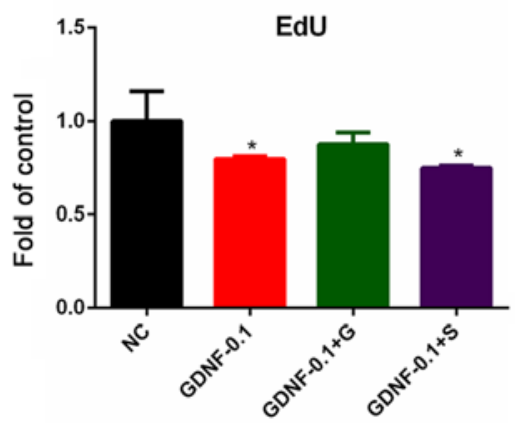

D

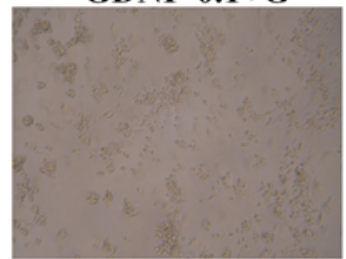

Hoechst
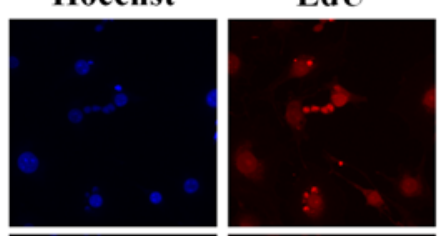

Merged

GDNF-0.1
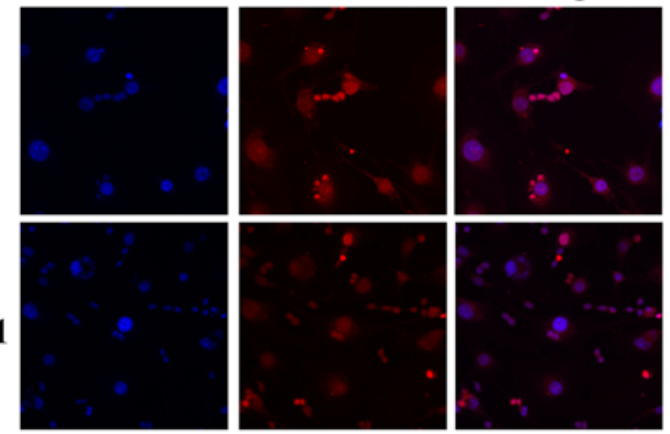

GDNF-0.1+G
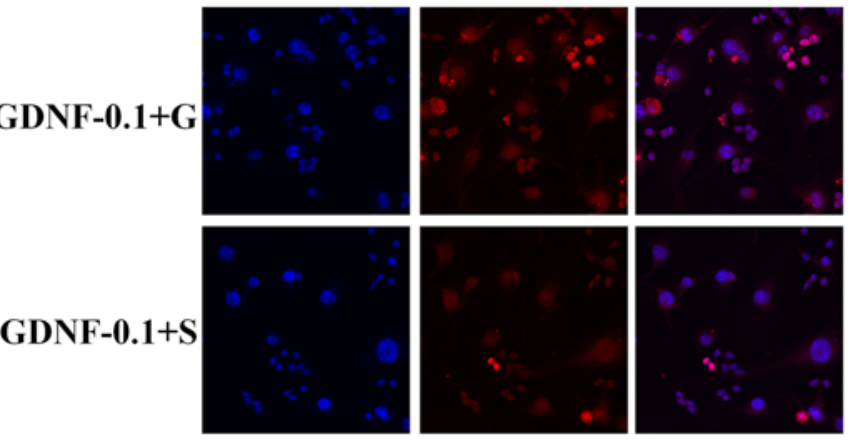
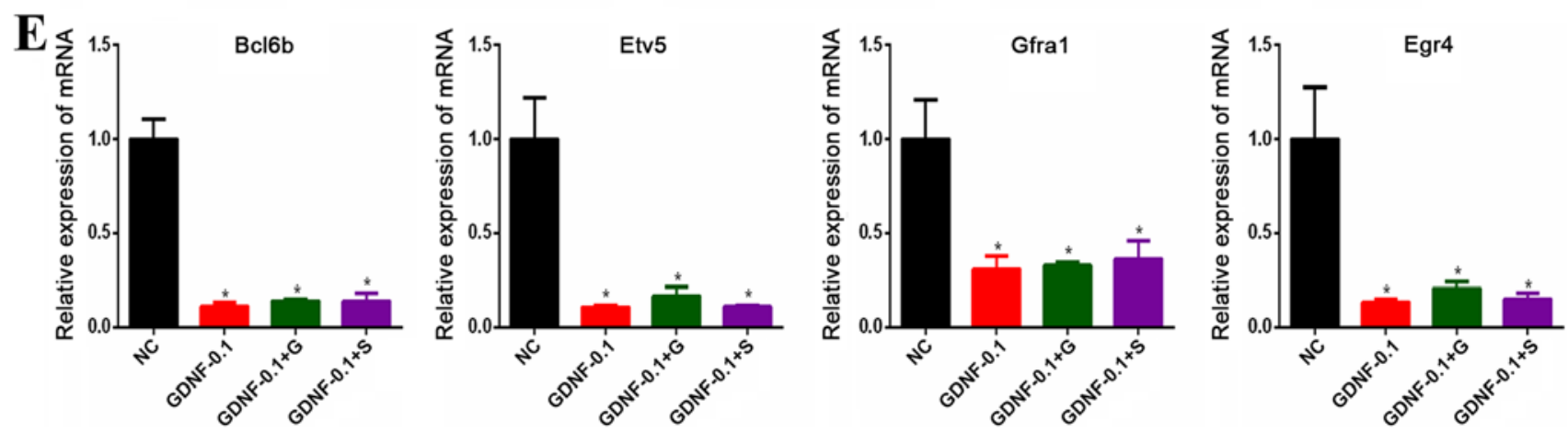

Figure 2. Effects of glycylglycine and sorbitol on the viability of SSCs. SSCs were exposed to complete medium (NC), GDNF (0.1 ng/ml), GDNF (0.1 ng/ml) and glycylglycine $(10 \mu \mathrm{M})$, or GDNF $(0.1 \mathrm{ng} / \mathrm{ml})$ and sorbitol $(10 \mu \mathrm{M})$. (A) Representative SSCs images under a light microscope on day 5 . Magnification, x10 objective. (B) Cell viability was determined by CCK-8 assay. (C) Relative cell proliferation, as assessed by EdU assay. (D) Staining for nuclei (blue) and cell proliferation (red). Images were acquired with the ArrayScan HCS Reader with a x20 objective. (E) The mRNA expression levels of self-renewal genes (Bcl6b, Etv5, Egr4 and Gfral) were detected by reverse transcription-quantitative polymerase chain reaction. "P<0.05 vs. respective NC. SSCs, spermatogonial stem cells; GDNF, glial cell line-derived neurotrophic factor; G, glycylglycine; S, sorbitol; CCK-8, Cell Counting Kit-8; NC, control; Bcl6b, B-cell CLL/lymphoma 6 member B; Etv5, ETS variant 5; Egr4, early growth response protein 4; Gfral, GDNF family receptor $\alpha 1$.

Glycylglycine rescues the GDNF-deprivation-induced inhibition of SSCs proliferation. The effects of glycylglycine and sorbitol on the proliferation of SSCs were investigated using a CCK-8 and an EdU assay. SSCs were cultured in either complete medium or media without GDNF, and treated with either glycylglycine or sorbitol. After 5 days of treatment, the SSCs were imaged using a light microscope (Fig. 2A). The CCK- 8 assay demonstrated that the viability of SSCs decreased significantly after GDNF deprivation. The addition of glycylglycine partially rescued the viability of the SSCs to levels similar to those observed in SSCs cultured in complete media, while sorbitol treatment failed to rescue their viability (Fig. 2B). Similar results were obtained with the EdU assay (Fig. 2C and D).

mRNA levels of self-renewal genes (Bcl6b, Etv5, Gfral and Egr4) remain unaltered following rescue with glycylglycine. The effects of glycylglycine and sorbitol on the expression of 
SSCs self-renewal genes was assessed via RT-qPCR analysis of Bcl6b, Etv5, Gfral and Egr4. The results demonstrated that GDNF deprivation downregulated the expression of the self-renewal genes of SSCs, and the addition of either glycylglycine or sorbitol did not restore the expression of these genes to normal levels (Fig. 2E).

\section{Discussion}

SSCs are responsible for accurately maintaining and transmitting parental genetic information (21). The proliferation and self-renewal of SSCs are precisely regulated by intrinsic and extrinsic signals (22). It is well known that GDNF serves a crucial role in regulating the proliferation of SSCs, and that the GDNF concentration is important for maintaining the function of SSCs (23). The present study demonstrated that GDNF deprivation decreased the viability of SSCs, and that this decrease in viability may be rescued by the addition of glycylglycine.

Glycylglycine is a dipeptide of glycine, which is the simplest amino acid. Glycine, a nonessential amino acid, is involved in the production of DNA, phospholipids and collagen, as well as the release of energy (24). It has also been demonstrated that glycine serves a key role in cell proliferation, potentially through the action of modifying enzymes $(25,26)$. The synthesizing enzymes serine hydroxymethyltransferase, cytosolic, serine hydroxymethyltransferase, mitochondrial, C-1-tetrahydrofolate synthase, cytoplasmic, and monofunctional C1-tetrahydrofolate synthase, mitochondrial are responsible for glycine synthesis (27). Glycylglycine, which is used as a buffer, is a starting template for the preparation of more complex peptides and a substrate for the enzyme glycylglycine dipeptidase (28). Glycylglycine has been utilized in the purification and characterization of a fructose-6-phosphate aldolase from Escherichia coli and in the characterization of a poly(L-malate) hydrolase from a strain of Comamonas acidovorans $(29,30)$. Glycylglycine has also been used in a [35S] guanosine triphosphate- $\gamma-\mathrm{S}$ binding assay to measure the functional coupling of $\mathrm{G}$ proteins with receptors (31). Sorbitol is a sugar alcohol that is gradually metabolized by the human body (32). Previous studies reported that sorbitol promotes proliferation in various cell types $(33,34)$. However, the results of the present study indicated that glycylglycine, rather than sorbitol, may be a crucial molecule in the GDNF-dependent proliferation of SSCs.

GDNF-induced cell signaling also serves a central role in the self-renewal of SSCs (21). Previous studies demonstrated that GDNF regulates self-renewal through different signaling pathways, including but not limited to Ras/extracellular signal-regulated kinases $1 / 2$ (35), mitogen-activated protein 2 kinase 1 (36) and phosphatidylinositol-4,5-bisphosphate 3-kinase/protein kinase B (37). Meanwhile, a number of GDNF-associated genes, including Gfral, Bcl $6 b$ and Etv5, have all been determined to be involved in the self-renewal of SSCs $(36,38)$. The present study demonstrated that GDNF deprivation downregulated the expression of these self-renewal genes in cultured SSCs, and that the addition of glycylglycine or sorbitol did not rescue their expression. Unlike what was observed in the proliferation of SSCs, these data suggested that glycylglycine is not involved in the self-renewal of SSCs.

Although there are various shared factors and pathways, the proliferation and self-renewal of SSCs are regulated differently (39). Proliferation is an important feature of life that contributes to development and growth via division. The self-renewal of SSCs is a unique form of cell division in which SSCs proliferate and differentiate. This process requires control of the cell cycle and maintenance of the undifferentiated state (40).

In conclusion, through unbiased metabolomic analyses, the present study identified that the production of glycylglycine and sorbitol were significantly altered following GDNF deprivation. While the addition of glycylglycine restored the impaired proliferation of SSCs, the addition of sorbitol had no effect, which suggests that glycylglycine serves an important role in the proliferation of SSCs. This study also provided novel insights into the mechanism underlying the proliferation of SSCs.

\section{Acknowledgements}

The authors would like to thank Dr Lufan Li (State Key Laboratory of Reproductive Medicine, Nanjing Medical University) for assistance with the mouse work.

\section{Funding}

The present study was supported by the National Natural Science Foundation (grant nos. 81602884 and 81602885); the Science and Technology Development Foundation of Nanjing Medical University (grant no. 2015NJMU006); and the Priority Academic Program Development of Jiangsu Higher Education Institutions.

\section{Availability of data and materials}

The datasets generated and/or analyzed in the present study are available from the corresponding author on reasonable request.

\section{Authors' contributions}

BX performed the EdU experiments and drafted the manuscript. XWe performed the experiments on spermatogonial stem cells. MC analyzed and interpreted the metabolomic data. ZH and TD performed the RT-qPCR experiments. KX and $\mathrm{WH}$ were involved in the design of the study and manuscript revisions. YZ, KZ and XH performed the Cell Counting Kit- 8 assay, and participated in metabolomic preparation and table preparation. XWu and YX made substantial contributions to the study conception and design. All authors read and approved the final manuscript.

\section{Ethics approval and consent to participate}

This study was approved by the Animal Ethical and Welfare Committee of Nanjing Medical University (Nanjing, China).

\section{Patient consent for publication}

Not applicable.

\section{Competing interests}

The authors declare that they have no competing interests. 


\section{References}

1. Phillips BT, Gassei K and Orwig KE: Spermatogonial stem cell regulation and spermatogenesis. Philos Trans R Soc Lond B Biol Sci 365:1663-1678, 2010.

2. Hofmann MC: Gdnf signaling pathways within the mammalian spermatogonial stem cell niche. Mol Cell Endocrinol 288: 95-103, 2008.

3. Meng X, Lindahl M, Hyvonen ME, Parvinen M, de Rooij DG, Hess MW, Raatikainen-Ahokas A, Sainio K, Rauvala H, Lakso M, et al: Regulation of cell fate decision of undifferentiated spermatogonia by GDNF. Science 287: 1489-1493, 2000.

4. Meng X, de Rooij DG, Westerdahl K, Saarma M and Sariola H: Promotion of seminomatous tumors by targeted overexpression of glial cell line-derived neurotrophic factor in mouse testis. Cancer Res 61: 3267-3271, 2001.

5. Hofmann MC, Braydich-Stolle L, Dettin L, Johnson E and Dym M: Immortalization of mouse germ line stem cells. Stem Cells 23: 200-210, 2005.

6. Kubota H, Avarbock MR and Brinster RL: Culture conditions and single growth factors affect fate determination of mouse spermatogonial stem cells. Biol Reprod 71: 722-731, 2004.

7. Horgan RP and Kenny LC: 'Omic'technologies: Genomics, transcriptomics, proteomics and metabolomics. Obstetrician Gynaecol 13: 189-195, 2011.

8. Ito K and Suda T: Metabolic requirements for the maintenance of self-renewing stem cells. Nat Rev Mol Cell Biol 15: 243-256, 2014.

9. Panopoulos AD, Yanes O, Ruiz S, Kida YS, Diep D, Tautenhahn R, Herrerias A, Batchelder EM, Plongthongkum N, Lutz M, et al: The metabolome of induced pluripotent stem cells reveals metabolic changes occurring in somatic cell reprogramming. Cell Res 22: 168-177, 2012.

10. Yanes O, Clark J, Wong DM, Patti GJ, Sanchez-Ruiz A, Benton HP, Trauger SA, Desponts C, Ding S and Siuzdak G Metabolic oxidation regulates embryonic stem cell differentiation. Nat Chem Biol 6: 411-417, 2010.

11. Xu B, Chen M, Ji X, Mao Z, Zhang X, Wang X and Xia Y: Metabolomic profiles delineate the potential role of glycine in gold nanorod-induced disruption of mitochondria and blood-testis barrier factors in TM-4 cells. Nanoscale 6: 8265-8273, 2014.

12. Shen H, Xu W, Zhang J, Chen M, Martin FL, Xia Y, Liu L, Dong $\mathrm{S}$ and Zhu YG: Urinary metabolic biomarkers link oxidative stress indicators associated with general arsenic exposure to male infertility in a han chinese population. Environ Sci Technol 47: 8843-8851,2013.

13. Kubota $\mathrm{H}$ and Brinster RL: Culture of rodent spermatogonial stem cells, male germline stem cells of the postnatal animal. Methods Cell Biol 86: 59-84, 2008.

14. Wei X, Jia Y, Xue Y, Geng L, Wang M, Li L, Wang M, Zhang X and Wu X: GDNF-expressing STO feeder layer supports the long-term propagation of undifferentiated mouse spermatogonia with stem cell properties. Sci Rep 6: 36779, 2016.

15. Xu B, Chen M, Ji X, Yao M, Mao Z, Zhou K, Xia Y, Han X and Tang W: Metabolomic profiles reveal key metabolic changes in heat stress-treated mouse Sertoli cells. Toxicol In Vitro 29: $1745-1752,2015$

16. Yuan B, Wu W, Chen M, Gu H, Tang Q, Guo D, Chen T, Chen Y, Lu C, Song L, et al: From the Cover: Metabolomics reveals a role of betaine in prenatal DBP exposure-induced epigenetic transgenerational failure of spermatogenesis in Rats. Toxicol Sci 158 : 356-366, 2017

17. Gika HG, Macpherson E, Theodoridis GA and Wilson ID: Evaluation of the repeatability of ultra-performance liquid chromatography-TOF-MS for global metabolic profiling of human urine samples. J Chromatogr B Analyt Technol Biomed Life Sci 871: 299-305, 2008.

18. L Eriksson, T Byrne, E Johansson, J Trygg, C Vikström: Multi-and megavariate data analysis basic principles and applications.

19. Cai Z,Zhao JS, Li JJ, Peng DN, Wang XY, Chen TL, Qiu YP, Chen PP, $\mathrm{Li} \mathrm{WJ}, \mathrm{Xu} \mathrm{LY}$, et al: A combined proteomics and metabolomics profiling of gastric cardia cancer reveals characteristic dysregulations in glucose metabolism. Mol Cell Proteomics 9: 2617-2628, 2010.

20. Livak KJ and Schmittgen TD: Analysis of relative gene expression data using real-time quantitative PCR and the 2(-Delta Delta C(T)) method. Methods 25: 402-408, 2001.

21. Kubota H, Avarbock MR and Brinster RL: Growth factors essential for self-renewal and expansion of mouse spermatogonial stem cells. Proc Natl Acad Sci USA 101: 16489-16494, 2004.
22. Mei XX, Wang $\mathrm{J}$ and $\mathrm{Wu} \mathrm{J}$ : Extrinsic and intrinsic factors controlling spermatogonial stem cell self-renewal and differentiation. Asian J Androl 17: 347-54, 2015.

23. Meng X, Lindahl M, Hyvönen ME, Parvinen M, de Rooij DG, Hess MW, Raatikainen-Ahokas A, Sainio K, Rauvala H, Lakso M, et al: Regulation of cell fate decision of undifferentiated spermatogonia by GDNF. Science 287: 1489-93, 2000

24. Cao M, Zhao L, Chen H, Xue W and Lin D: NMR-based metabolomic analysis of human bladder cancer. Anal Sci 28: 451-456, 2012.

25. Jain M, Nilsson R, Sharma S, Madhusudhan N, Kitami T, Souza AL, Kafri R, Kirschner MW, Clish CB and Mootha VK: Metabolite profiling identifies a key role for glycine in rapid cancer cell proliferation. Science 336: 1040-1044, 2012.

26. Ding J, Li T, Wang X, Zhao E, Choi JH, Yang L, Zha Y, Dong Z, Huang S, Asara JM, et al: The histone $\mathrm{H} 3$ methyltransferase G9A epigenetically activates the serine-glycine synthesis pathway to sustain cancer cell survival and proliferation. Cell Metab 18: 896-907, 2013.

27. Tedeschi PM, Markert EK, Gounder M, Lin H, Dvorzhinski D, Dolfi SC, Chan LL, Qiu J, DiPaola RS, Hirshfield KM, et al: Contribution of serine, folate and glycine metabolism to the ATP, NADPH and purine requirements of cancer cells. Cell Death Dis 4: e877, 2013.

28. Tsuboi KK, Penefsky ZJ and Hudson PB: Enzymes of the human erythrocyte. III. Tripeptidase, purification and specific properties. Arch Biochem Biophys 68: 54-68, 1957.

29. Gödde $C$, Liebergesell $M$ and Steinbüchel A: Isolation of poly(beta-L-malic acid)-degrading bacteria and purification and characterization of the PMA hydrolase from Comamonas acidovorans strain 7789. FEMS Microbiol Lett 173: 365-372, 1999.

30. Schneider S, Sandalova T, Schneider G, Sprenger GA and Samland AK: Replacement of a phenylalanine by a tyrosine in the active site confers fructose-6-phosphate aldolase activity to the transaldolase of Escherichia coli and human origin. J Biol Chem 283:30064-72, 2008.

31. Happe HK, Bylund DB and Murrin LC: Agonist-stimulated [35S] GTPgammaS autoradiography: Optimization for high sensitivity. Eur J Pharmacol 422: 1-13, 2001.

32. Kava R, Meister K and Kroger M: Low-calorie sweeteners and other sugar substitutes: A review of the safety issues. Comprehensive Rev Food Sci Food Safety 5: 35-47, 2006

33. Graier WF, Grubenthal I, Dittrich P, Wascher TC and Kostner GM: Intracellular mechanism of high D-glucose-induced modulation of vascular cell proliferation. Eur J Pharmacol 294: 221-229, 1995.

34. Turner JL and Bierman EL: Effects of glucose and sorbitol on proliferation of cultured human skin fibroblasts and arterial smooth-muscle cells. Diabetes 27: 583-588, 1978.

35. He Z, Jiang J, Kokkinaki M, Golestaneh N, Hofmann MC and Dym M: Gdnf upregulates c-Fos transcription via the Ras/Erk1/2 pathway to promote mouse spermatogonial stem cell proliferation. Stem Cells 26: 266-278, 2008.

36. Ishii K, Kanatsu-Shinohara M, Toyokuni S and Shinohara T: FGF2 mediates mouse spermatogonial stem cell self-renewal via upregulation of Etv5 and Bcl6b through MAP2K1 activation. Development 139: 1734-1743, 2012.

37. Wu X, Oatley JM, Oatley MJ, Kaucher AV, Avarbock MR and Brinster RL: The POU domain transcription factor POU3F1 is an important intrinsic regulator of GDNF-induced survival and self-renewal of mouse spermatogonial stem cells. Biol Reprod 82:1103-1111, 2010.

38. Wu X, Goodyear SM, Tobias JW, Avarbock MR and Brinster RL: Spermatogonial stem cell self-renewal requires ETV5-mediated downstream activation of Brachyury in mice. Biol Reprod 85: 1114-1123, 2011.

39. Puglisi MA, Tesori V, Lattanzi W, Gasbarrini GB and Gasbarrini A: Colon cancer stem cells: Controversies and perspectives. World J Gastroenterol 19: 2997-3006, 2013.

40. Chen T, Heller E, Beronja S, Oshimori N, Stokes N and Fuchs E: An RNA interference screen uncovers a new molecule in stem cell self-renewal and long-term regeneration. Nature 485: 104-108, 2012.

This work is licensed under a Creative Commons Attribution-NonCommercial-NoDerivatives 4.0 International (CC BY-NC-ND 4.0) License. 